\title{
Compactness in Metric Spaces
}

\author{
Kazuhisa Nakasho \\ Shinshu University \\ Nagano, Japan
}

\author{
Keiko Narita \\ Hirosaki-city \\ Aomori, Japan
}

\author{
Yasunari Shidama \\ Shinshu University \\ Nagano, Japan
}

\begin{abstract}
Summary. In this article, we mainly formalize in Mizar 2] the equivalence among a few compactness definitions of metric spaces, norm spaces, and the real line. In the first section, we formalized general topological properties of metric spaces. We discussed openness and closedness of subsets in metric spaces in terms of convergence of element sequences. In the second section, we firstly formalize the definition of sequentially compact, and then discuss the equivalence of compactness, countable compactness, sequential compactness, and totally boundedness with completeness in metric spaces.

In the third section, we discuss compactness in norm spaces. We formalize the equivalence of compactness and sequential compactness in norm space. In the fourth section, we formalize topological properties of the real line in terms of convergence of real number sequences. In the last section, we formalize the equivalence of compactness and sequential compactness in the real line. These formalizations are based on [20, [5, [17, 14], and [4].
\end{abstract}

MSC: 46B50 54E45 03B35

Keywords: metric spaces; normed linear spaces; compactness

MML identifier: TOPMETR4, version: 8.1.05 5.37.1275

\section{Topological Properties of Metric Spaces}

Now we state the propositions:

(1) Let us consider a non empty set $M$, and a sequence $x$ of $M$. Suppose $\operatorname{rng} x$ is finite. Then there exists an element $z$ of $M$ such that

(i) $x^{-1}(\{z\}) \subseteq \mathbb{N}$, and

(ii) $x^{-1}(\{z\})$ is infinite. 
Proof: Define $\mathcal{X}$ (object) $=x^{-1}\left(\left\{\$_{1}\right\}\right)$. Set $K=\{\mathcal{X}(w)$, where $w$ is an element of $M: w \in \operatorname{rng} x\}$. $K$ is finite from [18, Sch. 21]. For every set $Y$ such that $Y \in K$ holds $Y$ is finite. $\operatorname{dom} x \subseteq \cup K$ by [6, (3)].

(2) Let us consider a subset $X$ of $\mathbb{N}$. Suppose $X$ is infinite. Then there exists an increasing sequence $N$ of $\mathbb{N}$ such that $\operatorname{rng} N \subseteq X$.

Proof: Reconsider $B=2^{X}$ as a non empty set. Reconsider $N_{0}=\min ^{*} X$ as an element of $\mathbb{N}$. Reconsider $Y_{0}=X$ as an element of $B$. Define $\mathcal{P}$ [object, object, set, object, set] $\equiv \$_{5}=\$_{3} \backslash\left\{\$_{2}\right\}$ and $\$_{4}=\min ^{*} \$_{5}$. For every natural number $n$ and for every element $x$ of $\mathbb{N}$ and for every element $y$ of $B$, there exists an element $x_{1}$ of $\mathbb{N}$ and there exists an element $y_{1}$ of $B$ such that $\mathcal{P}\left[n, x, y, x_{1}, y_{1}\right]$. Consider $N$ being a sequence of $\mathbb{N}, Y$ being a sequence of $B$ such that $N(0)=N_{0}$ and $Y(0)=Y_{0}$ and for every natural number $n, \mathcal{P}[n, N(n), Y(n), N(n+1), Y(n+1)]$ from [13, Sch. 3]. Define $\mathcal{Q}$ [natural number] $\equiv N\left(\$_{1}\right)=\min ^{*}\left(Y\left(\$_{1}\right)\right)$ and $N\left(\$_{1}\right) \in Y\left(\$_{1}\right)$ and $Y\left(\$_{1}\right)$ is infinite and $Y\left(\$_{1}\right) \subseteq \mathbb{N}$. For every natural number $i$ such that $\mathcal{Q}[i]$ holds $\mathcal{Q}[i+1]$ by [8, (31)]. For every natural number $i, \mathcal{Q}[i]$ from [1, Sch. 2]. $\operatorname{rng} N \subseteq X$ by [7, (11)]. For every natural number $i, N(i)<N(i+1)$.

(3) Let us consider a non empty metric space $M$, and a subset $V$ of $M_{\text {top }}$. Suppose $V$ is open. Then there exists a family $F$ of subsets of $M$ such that

(i) $F=\{\operatorname{Ball}(x, r)$, where $x$ is an element of $M, r$ is a real number $: r>$ 0 and $\operatorname{Ball}(x, r) \subseteq V\}$, and

(ii) $V=\bigcup F$.

Proof: Set $F=\{\operatorname{Ball}(x, r)$, where $x$ is an element of $M, r$ is a real number: $r>0$ and $\operatorname{Ball}(x, r) \subseteq V\}$. For every object $z$ such that $z \in F$ holds $z \in$ the open set family of $M$ by [3, (29)]. Reconsider $Q=\bigcup F$ as a subset of $M_{\text {top }}$. For every object $z, z \in V$ iff $z \in Q$ by [9, (15)], [12, (1), (11)].

(4) Let us consider a non empty metric space $M$, a subset $X$ of $M_{\text {top }}$, and an element $p$ of $M$. Then $p \in \bar{X}$ if and only if for every real number $r$ such that $0<r$ holds $X$ meets $\operatorname{Ball}(p, r)$.

(5) Let us consider a non empty metric space $M$, a subset $X$ of $M_{\text {top }}$, and an object $x$. Then $x \in \bar{X}$ if and only if there exists a sequence $S$ of $M$ such that for every natural number $n, S(n) \in X$ and $S$ is convergent and $\lim S=x$.

(6) Let us consider a non empty metric space $M$, and a subset $X$ of $M_{\text {top }}$. Then $X$ is closed if and only if for every sequence $S$ of $M$ such that for every natural number $n, S(n) \in X$ and $S$ is convergent holds $\lim S \in X$. The theorem is a consequence of (5). 
(7) Let us consider non empty metric spaces $X, Y$, and a function $f$ from $X_{\text {top }}$ into $Y_{\text {top. }}$. Then $f$ is continuous if and only if for every sequence $S$ of $X$ and for every sequence $T$ of $Y$ such that $S$ is convergent and $T=f \cdot S$ holds $T$ is convergent and $\lim T=f(\lim S)$.

Proof: For every subset $B$ of $Y_{\text {top }}$ such that $B$ is closed holds $f^{-1}(B)$ is closed by [7, (15)], (6).

\section{Compactness in Metric Spaces}

Let $M$ be a non empty metric space and $X$ be a subset of $M$. We say that $X$ is sequentially compact if and only if

(Def. 1) for every sequence $S_{1}$ of $M$ such that $\operatorname{rng} S_{1} \subseteq X$ there exists a sequence $S_{2}$ of $M$ such that there exists an increasing sequence $N$ of $\mathbb{N}$ such that $S_{2}=S_{1} \cdot N$ and $S_{2}$ is convergent and $\lim S_{2} \in X$.

Let us observe that every subset of $M$ which is empty is also sequentially compact.

We say that $M$ is sequentially compact if and only if

(Def. 2) $\Omega_{M}$ is sequentially compact.

Now we state the proposition:

(8) Let us consider a non empty metric space $M$, a subset $X$ of $M$, a subset $Y$ of $M_{\mathrm{top}}$, an element $x$ of $M$, and an element $y$ of $M_{\mathrm{top}}$. Suppose $X=Y$ and $x=y$. Then $y$ is an accumulation point of $Y$ if and only if for every real number $r$ such that $0<r$ holds $\operatorname{Ball}(x, r)$ meets $X \backslash\{x\}$.

Let us consider a non empty metric space $M$. Now we state the propositions:

(9) If $M_{\text {top }}$ is countably-compact, then $M$ is sequentially compact.

ProOF: For every subset $X$ of $M$ such that $X$ is infinite there exists an element $x$ of $M$ such that for every real number $r$ such that $0<r$ holds $\operatorname{Ball}(x, r)$ meets $X \backslash\{x\}$ by [16, (28)], [11, (16)], (8). For every sequence $x$ of $M$ such that $\operatorname{rng} x \subseteq \Omega_{M}$ there exists a sequence $x_{1}$ of $M$ such that there exists an increasing sequence $N$ of $\mathbb{N}$ such that $x_{1}=x \cdot N$ and $x_{1}$ is convergent and $\lim x_{1} \in \Omega_{M}$ by (1), (2), [7, (4), (38), (15)].

(10) If $M$ is sequentially compact, then $M_{\text {top }}$ is countably-compact.

Proof: For every subset $X$ of $M$ such that $X$ is infinite there exists an element $x$ of $M$ such that for every real number $r$ such that $0<r$ holds $\operatorname{Ball}(x, r)$ meets $X \backslash\{x\}$ by [15, (3)], [7, (2)], [19, (26)], [7, (112)]. For every subset $A$ of $M_{\text {top }}$ such that $A$ is infinite holds $\operatorname{Der} A$ is not empty by $(8),[11,(16)]$.

(11) $M_{\text {top }}$ is compact if and only if $M$ is sequentially compact. The theorem is a consequence of $(9)$. 
(12) $M$ is totally bounded and complete if and only if $M$ is sequentially compact. The theorem is a consequence of (11).

Let us consider a non empty metric space $M$ and a non empty subset $S$ of $M$. Now we state the propositions:

(13) $S$ is sequentially compact if and only if $M\lceil S$ is sequentially compact. Proof: For every sequence $S_{1}$ of $M$ such that $\operatorname{rng} S_{1} \subseteq S$ there exists a sequence $S_{2}$ of $M$ such that there exists an increasing sequence $N$ of $\mathbb{N}$ such that $S_{2}=S_{1} \cdot N$ and $S_{2}$ is convergent and $\lim S_{2} \in S$ by [7, (6)].

(14) $S$ is sequentially compact if and only if $M \uparrow S$ is compact. The theorem is a consequence of (11) and (13).

(15) Let us consider a non empty metric space $M$, a subset $S$ of $M$, and a subset $T$ of $M_{\text {top }}$. If $T=S$, then $T$ is compact iff $S$ is sequentially compact. The theorem is a consequence of (11) and (13).

(16) Let us consider a non empty metric space $M$, a non empty subset $S$ of $M$, and a non empty subset $T$ of $M_{\mathrm{top}}$. Suppose $T=S$. Then $M_{\mathrm{top}}\lceil T$ is countably-compact if and only if $S$ is sequentially compact. The theorem is a consequence of (11) and (13).

(17) Let us consider a non empty metric space $M$, and a non empty subset $S$ of $M$. Then $M\lceil S$ is totally bounded and complete if and only if $S$ is sequentially compact. The theorem is a consequence of (12) and (13).

\section{Compactness in Norm Spaces}

Now we state the propositions:

(18) Let us consider a real normed space $N$, a subset $S$ of $N$, and a subset $T$ of MetricSpaceNorm $N$. If $S=T$, then $S$ is compact iff $T$ is sequentially compact.

(19) Let us consider a real normed space $N$, a subset $S$ of $N$, and a subset $T$ of TopSpaceNorm $N$. If $S=T$, then $S$ is compact iff $T$ is compact. The theorem is a consequence of (15) and (18).

\section{Topological Properties of the Real Line}

Let us consider a sequence $S_{1}$ of the metric space of real numbers, a sequence $s$ of real numbers, a real number $g$, and an element $g_{1}$ of the metric space of real numbers. Now we state the propositions:

(20) Suppose $S_{1}=s$ and $g=g_{1}$. Then for every real number $p$ such that $0<p$ there exists a natural number $n$ such that for every natural number 
$m$ such that $n \leqslant m$ holds $|s(m)-g|<p$ if and only if for every real number $p$ such that $0<p$ there exists a natural number $n$ such that for every natural number $m$ such that $n \leqslant m$ holds $\rho\left(S_{1}(m), g_{1}\right)<p$.

PROOF: For every real number $p$ such that $0<p$ there exists a natural number $n$ such that for every natural number $m$ such that $n \leqslant m$ holds $|s(m)-g|<p$ by [9, (11)].

(21) If $S_{1}=s$ and $g=g_{1}$, then $s$ is convergent and $\lim s=g$ iff $S_{1}$ is convergent and $\lim S_{1}=g_{1}$. The theorem is a consequence of (20).

(22) Let us consider a sequence $S_{1}$ of the metric space of real numbers, and a sequence $s$ of real numbers. Suppose $S_{1}=s$ and $s$ is convergent. Then

(i) $S_{1}$ is convergent, and

(ii) $\lim S_{1}=\lim s$.

The theorem is a consequence of (20).

\section{Compactness in the Real Line}

Now we state the propositions:

(23) Let us consider a subset $N$ of $\mathbb{R}$, and a subset $M$ of $\mathbb{R}^{\mathbf{1}}$. Suppose $N=M$. Then for every family $F$ of subsets of $\mathbb{R}$ such that $F$ is a cover of $N$ and for every subset $P$ of $\mathbb{R}$ such that $P \in F$ holds $P$ is open there exists a family $G$ of subsets of $\mathbb{R}$ such that $G \subseteq F$ and $G$ is cover of $N$ and finite if and only if for every family $F_{1}$ of subsets of $\mathbb{R}^{\mathbf{1}}$ such that $F_{1}$ is cover of $M$ and open there exists a family $G_{1}$ of subsets of $\mathbb{R}^{\mathbf{1}}$ such that $G_{1} \subseteq F_{1}$ and $G_{1}$ is cover of $M$ and finite.

Proof: Reconsider $F_{1}=F$ as a family of subsets of $\mathbb{R}^{\mathbf{1}}$. For every subset $P_{1}$ of $\mathbb{R}^{1}$ such that $P_{1} \in F_{1}$ holds $P_{1}$ is open by [10, (39)]. Consider $G_{1}$ being a family of subsets of $\mathbb{R}^{\mathbf{1}}$ such that $G_{1} \subseteq F_{1}$ and $G_{1}$ is cover of $M$ and finite.

(24) Let us consider a subset $N$ of $\mathbb{R}$. Then $N$ is compact if and only if for every family $F$ of subsets of $\mathbb{R}$ such that $F$ is a cover of $N$ and for every subset $P$ of $\mathbb{R}$ such that $P \in F$ holds $P$ is open there exists a family $G$ of subsets of $\mathbb{R}$ such that $G \subseteq F$ and $G$ is cover of $N$ and finite. The theorem is a consequence of (23). 


\section{REFERENCES}

[1] Grzegorz Bancerek. The fundamental properties of natural numbers Formalized Mathematics, 1(1):41-46, 1990.

[2] Grzegorz Bancerek, Czesław Byliński, Adam Grabowski, Artur Korniłowicz, Roman Matuszewski, Adam Naumowicz, Karol Pąk, and Josef Urban. Mizar: State-of-the-art and beyond In Manfred Kerber, Jacques Carette, Cezary Kaliszyk, Florian Rabe, and Volker Sorge, editors, Intelligent Computer Mathematics, volume 9150 of Lecture Notes in Computer Science, pages 261-279. Springer International Publishing, 2015. ISBN 978-3319-20614-1. doi:10.1007/978-3-319-20615-8_17.

[3] Leszek Borys. Paracompact and metrizable spaces. Formalized Mathematics, 2(4):481485, 1991.

[4] Nicolas Bourbaki. General Topology: Chapters 1-4. Springer Science and Business Media, 2013.

[5] Nicolas Bourbaki, H.G. Eggleston, and S. Madan. Elements of mathematics: Topological vector spaces. Springer-Verlag, 1987.

[6] Czesław Byliński. Functions and their basic properties Formalized Mathematics, 1(1): 55-65, 1990.

[7] Czesław Byliński. Functions from a set to a set Formalized Mathematics, 1(1):153-164, 1990.

[8] Czesław Byliński. Some basic properties of sets Formalized Mathematics, 1(1):47-53, 1990.

[9] Agata Darmochwał and Yatsuka Nakamura. Metric spaces as topological spaces - fundamental concepts Formalized Mathematics, 2(4):605-608, 1991.

[10] Adam Grabowski. On the subcontinua of a real line Formalized Mathematics, 11(3): 313-322, 2003.

[11] Adam Grabowski. On the boundary and derivative of a set Formalized Mathematics, 13 (1):139-146, 2005.

[12] Stanisława Kanas, Adam Lecko, and Mariusz Startek. Metric spaces Formalized Mathematics, 1(3):607-610, 1990.

[13] Artur Korniłowicz. Recursive definitions. Part II. Formalized Mathematics, 12(2):167$172,2004$.

[14] Erwin Kreyszig. Introductory Functional Analysis with Applications. Wiley, 1 edition, 1989.

[15] Gilbert Lee and Piotr Rudnicki. Dickson's lemma Formalized Mathematics, 10(1):29-37, 2002.

[16] Karol Pak. Complete spaces. Formalized Mathematics, 16(1):35-43, 2008. doi: $10.2478 / \mathrm{v} 10037-008-0006-2$

[17] Walter Rudin. Functional Analysis. New York, McGraw-Hill, 2nd edition, 1991.

[18] Andrzej Trybulec. Function domains and Frænkel operator Formalized Mathematics, 1 (3):495-500, 1990.

[19] Edmund Woronowicz. Relations and their basic properties Formalized Mathematics, 1 (1):73-83, 1990.

[20] Kosaku Yoshida. Functional Analysis. Springer, 1980.

Received June 30, 2016 